\title{
Gap Functions and Error Bounds for Variational-Hemivariational Inequalities
}

\author{
Nguyen Van Hung ${ }^{1,2}$ • Stanislaw Migórski ${ }^{3,4,5}$. \\ Vo Minh Tam $^{6} \cdot$ Shengda Zeng ${ }^{4,5}$ iD
}

Received: 30 October 2019 / Accepted: 19 February 2020 / Published online: 26 February 2020

(C) The Author(s) 2020

\begin{abstract}
In this paper we investigate the gap functions and regularized gap functions for a class of variational-hemivariational inequalities of elliptic type. First, based on regularized gap functions introduced by Yamashita and Fukushima, we establish some regularized gap functions for the variational-hemivariational inequalities. Then, the global error bounds for such inequalities in terms of regularized gap functions are derived by using the properties of the Clarke generalized gradient. Finally, an application to a stationary nonsmooth semipermeability problem is given to illustrate our main results.
\end{abstract}

Keywords Variational-hemivariational inequality · Gap function · Global error bound . Semipermeability problem

Mathematics Subject Classification 47J20 - 49J40 - 49J45 - 74M10 · 74M15

S. Zeng

zengshengda@163.com

N.V. Hung

nguyenvanhung2@tdtu.edu.vn

S. Migórski

stanislaw.migorski@uj.edu.pl

V.M. Tam

vmtam@dthu.edu.vn

1 Department for Management of Science and Technology Development, Ton Duc Thang University, Ho Chi Minh City, Vietnam

2 Faculty of Mathematics and Statistics, Ton Duc Thang University, Ho Chi Minh City, Vietnam

3 College of Applied Mathematics, Chengdu University of Information Technology, Chengdu, 610225, Sichuan Province, P.R. China

4 Guangxi Colleges and Universities Key Laboratory of Complex System Optimization and Big Data Processing, Yulin Normal University, Yulin 537000, P.R. China

5 Faculty of Mathematics and Computer Science, Jagiellonian University in Krakow ul. Lojasiewicza 6, 30348 Krakow, Poland

6 Department of Mathematics, Dong Thap University, Cao Lanh, Dong Thap, Vietnam 


\section{Introduction}

In the study of various complementarity and equilibrium problems occurring in operation research, economics, mechanics, mathematical programming, etc., we often naturally meet the variational inequality problem of the form:

$$
\text { find } u^{*} \in K \text { such that }\left\langle A u^{*}, v-u^{*}\right\rangle_{X} \geq 0 \text { for all } v \in K \text {. }
$$

Here $K$ is a nonempty closed convex subset of a normed space $X$ representing constraints, $A: X \rightarrow X^{*}$ is a given operator, and $\langle\cdot, \cdot\rangle_{X}$ denotes the duality pairing between $X$ and its dual $X^{*}$. Among several approaches available in the literature, it is well known that the variational inequality (1.1) can be solved by transforming it into an equivalent optimization problem for the so-called merit function $\pi(\cdot ; \alpha): X \rightarrow \mathbb{R} \cup\{+\infty\}$ defined by

$$
\pi(u ; \alpha)=\sup \left\{\langle A u, u-w\rangle_{X}-\alpha\|u-w\|_{X}^{2} \mid w \in K\right\} \quad \text { for } u \in K,
$$

where $\alpha$ is a nonnegative parameter. If $X$ is finite dimensional, this function was first introduced by Auslender in [4] for $\alpha=0$, and by Fukushima in [12] for $\alpha>0$. The function $\pi(\cdot ; 0)$ is usually called the gap function, and the function $\pi(\cdot ; \alpha)$ for $\alpha>0$ is called the regularized gap function.

It is known, see $[4,19]$, that for all $\alpha>0$, the function $\pi(\cdot ; \alpha)$ is nonnegative on $K$, and $\pi\left(u^{*} ; \alpha\right)=0$ whenever $u^{*}$ satisfies the variational inequality (1.1). An advantage of this approach is that the resulting optimization problem can be solved by descent algorithms which enjoys a global convergence property. However, it turns out that even for finite dimensional space $X$, the gap function fails to be differentiable in general, and it may not be finite valued. In contrast, the regularized gap function for $\alpha>0$ is nicer since it is finite valued and is differentiable whenever $A$ is differentiable, see [12] for details.

The gap functions are today very useful to investigate existence conditions, solution methods and stability conditions for optimization-related problems in order to simplify the computational aspects. Based on the idea of Fukushima [12] the regularized function of the Moreau-Yosida type has been developed by Yamashita and Fukushima in [49]. They also proposed the so-called error bounds for variational inequalities via the regularized gap functions. The notion of error bounds is known as an upper estimate of the distance between an arbitrary feasible point and the solution set of a certain problem. Such error estimates have played a vital role in convergence analysis of iterative algorithms for solving variational inequalities. In recent years, there have been many studies on gap functions for different models on different topics such as iterative algorithms [23], the Painlevé-Kuratowski convergence [2], stability of solutions [3, 20-22] and error bounds [6, 13, 24-26]. We also refer the reader to $[1,5,7,11,14,27-29]$ and the references therein for a more detailed discussion of interesting topic.

On the other hand, the theory of variational-hemivariational inequalities is known as a generalization of variational inequalities and hemivariational inequalities to the case involving both the convex and the nonconvex potentials, and based on the notion of the Clarke generalized gradient for locally Lipschitz functions. Interest in the study of variationalhemivariational inequalities was originally motivated by various problems in mechanics, see e.g., $[45,46]$. The theory of variational-hemivariational inequalities has been extensively studied by many authors in different directions, and it has found various applications in mechanics, engineering, especially in optimization and nonsmooth analysis. Recent existence results for variational-hemivariational inequalities can be found, in e.g., [16, 31, 
33-41, 43, 47, 48], the stability in the sense of convergence and the well-posedness, in e.g., [18, 30, 32, 50-53], and the computational issues have been addressed in, e.g., [15, 17].

To the best of our knowledge, up to now, there has not been any study on the gap functions and global error bounds for the variational-hemivariational inequalities. Our goal is to fill in this gap and provide new results in this area. The novelties of the paper are as follows. First, we introduce the gap functions and regularized gap functions for a class of variational-hemivariational inequalities. Also, we treat the gap functions for the Minty version of these inequalities. Next, we study the Moreau-Yosida regularized gap functions, introduced by Yamashita and Fukushima in [49], and provide two new global error bounds for variational-hemivariational inequalities via the regularized and the Moreau-Yosida regularized gap functions. Finally, we illustrate the abstract results by an application to a nonsmooth semipermeability obstacle problem described by an elliptic variational-hemivariational inequality for which we deliver global error bounds.

The article is arranged as follows. In Sect. 2, we recall basic definitions and results which are needed in the sequel and revisit the constrained variational-hemivariational inequality of elliptic type. In Sect. 3, we study some regularized gap functions of Yamashita-Fukushima type, and establish global error bounds for the variational-hemivariational inequalities. An application to a semipermeability problem for stationary heat problem is given in Sect. 4 to illustrate our main theoretical findings.

\section{Preliminaries}

In this section we recall the notation and some preliminary material which will be needed in the sequel. For more details, we refer to [8-10, 42].

Let $\left(X,\|\cdot\|_{X}\right)$ be a real Banach space with the dual $X^{*}$, and we denote by $\langle\cdot, \cdot\rangle_{X}$ the duality pairing between $X^{*}$ and $X$. We begin with the following definitions.

Definition 2.1 ((d)) A function $h: X \rightarrow \overline{\mathbb{R}}:=\mathbb{R} \cup\{+\infty\}$ is said to be

(a) proper, if $h \neq \equiv+\infty$.

(b) convex, if $h(t u+(1-t) v) \leq t h(u)+(1-t) h(v)$ for all $u, v \in X$ and $t \in[0,1]$.

(c) lower semicontinuous (1.s.c.) at $u \in X$, if for any sequence $\left\{u_{n}\right\} \subset X$ such that $u_{n} \rightarrow u$, it holds $h(u) \leq \liminf h\left(u_{n}\right)$.

(d) upper semicontinuous (u.s.c.) at $u \in X$, if for any sequence $\left\{u_{n}\right\} \subset X$ such that $u_{n} \rightarrow u$, it holds $\lim \sup h\left(u_{n}\right) \leq h(u)$.

(e) 1.s.c (resp. u.s.c.) on $X$, if $h$ is l.s.c (resp. u.s.c.) at every $u \in X$.

Definition 2.2 Let $f: X \rightarrow \overline{\mathbb{R}}$ be a proper, convex and 1.s.c. function. The convex subdifferential $\partial_{c} f: X \rightrightarrows X^{*}$ of $f$ is defined by

$$
\partial_{c} f(u)=\left\{u^{*} \in X^{*} \mid\left\langle u^{*}, v-u\right\rangle_{X} \leq f(v)-f(u) \text { for all } v \in X\right\} \text { for all } u \in X .
$$

An element $u^{*} \in \partial_{c} f(u)$ is called a subgradient of $f$ at $u \in X$.

Definition 2.3 A function $h: X \rightarrow \mathbb{R}$ is said to be locally Lipschitz, if for every $u \in X$, there exist a neighbourhood $U$ of $u$ and a constant $L_{u}>0$ such that

$$
\left|h\left(w_{1}\right)-h\left(w_{2}\right)\right| \leq L_{u}\left\|w_{1}-w_{2}\right\|_{X} \text { for all } w_{1}, w_{2} \in U .
$$


Given a locally Lipschitz function $h: X \rightarrow \mathbb{R}$, we denote by $h^{0}(u ; v)$ the Clarke generalized directional derivative of $h$ at the point $u \in X$ in the direction $v \in X$ defined by

$$
h^{0}(u ; v):=\limsup _{w \rightarrow u, t \rightarrow 0^{+}} \frac{h(w+t v)-h(w)}{t} .
$$

The generalized gradient of $h$ at $u \in X$, denoted by $\partial h(u)$, is a subset of $X^{*}$ given by

$$
\partial h(u)=\left\{u^{*} \in X^{*} \mid h^{0}(u ; v) \geq\left\langle u^{*}, v\right\rangle_{X} \text { for all } v \in X\right\} .
$$

The generalized directional derivative and generalized gradient of a locally Lipschitz function enjoy many nice properties and rich calculus. Below we collect some basic and useful results, see, e.g., [42, Proposition 3.23].

Lemma 2.1 Let $X$ be a real Banach space and $h: X \rightarrow \mathbb{R}$ be a locally Lipschitz function. Then the following assertions hold.

(a) For each $u \in X$, the function $X \ni v \mapsto h^{0}(u ; v) \in \mathbb{R}$ is finite, positively homogeneous and subadditive, and satisfies $\left|h^{0}(u ; v)\right| \leq L_{u}\|v\|_{X}$ for all $v \in X$, where $L_{u}>0$ is the Lipschitz constant of $h$ near $u$.

(b) The function $X \times X \ni(u, v) \mapsto h^{0}(u ; v) \in \mathbb{R}$ is upper semicontinuous.

(c) For every $u, v \in X$, it holds $h^{0}(u ; v)=\max \left\{\langle\xi, v\rangle_{X} \mid \xi \in \partial h(u)\right\}$.

Recall that a single-valued operator $A: X \rightarrow X^{*}$ is said to be pseudomonotone, if $A$ is a bounded operator and for every sequence $\left\{x_{n}\right\} \subseteq X$ converging weakly to $x \in X$ such that $\lim \sup \left\langle A x_{n}, x_{n}-x\right\rangle \leq 0$, we have $\langle A x, x-y\rangle \leq \liminf \left\langle A x_{n}, x_{n}-y\right\rangle$ for all $y \in X$.

Let $X$ be a reflexive Banach space and $K$ be a nonempty subset of $X$. Given an operator $A: K \rightarrow X^{*}$, functions $\varphi: K \times K \rightarrow \mathbb{R}$ and $J: X \rightarrow \mathbb{R}$, and $f \in X^{*}$, we are concerned with the study of the following constrained variational-hemivariational inequality.

Problem 2.1 Find $u \in K$ such that

$$
\langle A u-f, v-u\rangle_{X}+\varphi(u, v)-\varphi(u, u)+J^{0}(u ; v-u) \geq 0
$$

for all $v \in K$.

We now impose the following hypotheses on the data of Problem 2.1.

$A: X \rightarrow X^{*}$ is such that

(a) $A$ is pseudomonotone.

(b) $A$ is strongly monotone, i.e., there exists $m_{A}>0$ such that

$$
\left\langle A v_{1}-A v_{2}, v_{1}-v_{2}\right\rangle_{X} \geq m_{A}\left\|v_{1}-v_{2}\right\|_{X}^{2}
$$

for all $v_{1}, v_{2} \in X$. 
$\varphi: K \times K \rightarrow \mathbb{R}$ is such that

(a) for each $u \in K, \varphi(u, \cdot): K \rightarrow \mathbb{R}$ is convex and lower semicontinuous.

(b) there exists $\alpha_{\varphi}>0$ such that

$$
\varphi\left(u_{1}, v_{2}\right)-\varphi\left(u_{1}, v_{1}\right)+\varphi\left(u_{2}, v_{1}\right)-\varphi\left(u_{2}, v_{2}\right) \leq \alpha_{\varphi}\left\|u_{1}-u_{2}\right\|_{X}\left\|v_{1}-v_{2}\right\|_{X}
$$

for all $u_{1}, u_{2}, v_{1}, v_{2} \in K$.

$$
\left\{\begin{array}{l}
J: X \rightarrow \mathbb{R} \text { is a locally Lipschitz function such that } \\
\text { (a) }\|\partial J(v)\|_{X^{*}} \leq c_{0}+c_{1}\|v\|_{X} \quad \text { for all } v \in X \text { with some } c_{0}, c_{1} \geq 0 . \\
\text { (b) there exists } m_{J} \geq 0 \text { such that } \\
\quad J^{0}\left(v_{1} ; v_{2}-v_{1}\right)+J^{0}\left(v_{2} ; v_{1}-v_{2}\right) \leq m_{J}\left\|v_{1}-v_{2}\right\|_{X}^{2} \\
\text { for all } v_{1}, v_{2} \in K
\end{array}\right.
$$

$K$ is nonempty, closed and convex subset of $X$, and $f \in X^{*}$.

Remark 2.1 Note that in some recent works, such as [43, 47, 50], the authors have supposed that $A: X \rightarrow X^{*}$ and $J: X \rightarrow \mathbb{R}$ enjoy hypotheses (2.1) and (2.3). They also required the following additional condition

$$
\left\{\begin{array}{c}
\text { there exist } \alpha_{A}>0, \beta_{A}, \gamma_{A} \in \mathbb{R} \text { and } u_{0} \in K \text { such that } \\
\left\langle A v, v-u_{0}\right\rangle_{X} \geq \alpha_{A}\|v\|_{X}^{2}+\beta_{A}\|v\|_{X}+\gamma_{A}
\end{array}\right.
$$

where $\alpha_{A}>m_{J}$. However, this assumptions is redundant. Indeed, given $u_{0} \in K$, from hypotheses (2.1), we obtain

$$
\left\langle A v, v-u_{0}\right\rangle_{X} \geq m_{A}\|v\|_{X}^{2}-\left\|A u_{0}\right\|_{X^{*}}\|v\|_{X}-\left\|A u_{0}\right\|_{X^{*}}\left\|u_{0}\right\|_{X} \quad \text { for all } v \in X .
$$

It is obvious that the above estimate guarantees the condition (2.5).

We have the following existence and uniqueness result for Problem 2.1.

Theorem 2.1 Assume that (2.1)-(2.4) hold. If, in addition, the following smallness condition is satisfied

$$
\alpha_{\varphi}+m_{J}<m_{A},
$$

then Problem 2.1 has a unique solution. Moreover, $u$ solves Problem 2.1 if and only if it solves the following Minty variational-hemivariational inequality: find $u \in K$ such that

$$
\langle A v-f, v-u\rangle_{X}+\varphi(u, v)-\varphi(u, u)+J^{0}(v ; v-u) \geq 0
$$

for all $v \in K$.

Proof The existence and uniqueness of solution to Problem 2.1 is a direct consequence of [47, Theorem 1]. 
Let $u \in K$ be the unique solution to Problem 2.1. First, we note that the hypothesis (2.3)(b) is equivalent to the following relaxed monotonicity condition of the generalized gradient

$$
\langle\partial J(v)-\partial J(u), v-u\rangle_{X} \geq-m_{J}\|v-u\|_{X}^{2}
$$

for all $v, u \in X$. Next, the smallness condition (2.7) together with (2.9) and the strong monotonicity of $A$ implies

$$
\langle A v-A u, v-u\rangle_{X}+\left\langle\xi_{v}-\xi_{u}, v-u\right\rangle_{X} \geq\left(m_{A}-m_{J}\right)\|v-u\|_{X}^{2}
$$

for all $\xi_{v} \in \partial J(v), \xi_{u} \in \partial J(u)$, and all $u, v \in K$. Let $v \in K$ be arbitrary. Combining the above inequality, Lemma 2.1(c) and the definition of generalized gradient entails

$$
\begin{aligned}
\langle A v & -f, v-u\rangle_{X}+\varphi(u, v)-\varphi(u, u)+J^{0}(v ; v-u) \\
& \geq\left\langle A v-f+\xi_{v}, v-u\right\rangle_{X}+\varphi(u, v)-\varphi(u, u) \\
& \geq\left\langle A u-f+\xi_{u}, v-u\right\rangle_{X}+\varphi(u, v)-\varphi(u, u)+\left(m_{A}-m_{J}\right)\|v-u\|_{X}^{2} \\
& \geq\left\langle A u-f+\xi_{u}, v-u\right\rangle_{X}+\varphi(u, v)-\varphi(u, u) \\
& =\langle A u-f, v-u\rangle_{X}+\varphi(u, v)-\varphi(u, u)+J^{0}(u ; v-u) \geq 0
\end{aligned}
$$

for all $\xi_{v} \in \partial J(v)$, where $\xi_{u} \in \partial J(u)$ is such that

$$
J^{0}(u ; v-u)=\left\langle\xi_{u}, v-u\right\rangle_{X} .
$$

Since $v \in K$ is arbitrary, therefore, $u \in K$ solves the problem (2.8) too.

Conversely, let $u \in K$ be a solution to the problem (2.8). For any $v \in K$ and $t \in(0,1)$, we denote $v_{t}:=t v+(1-t) u \in K$. Inserting $v_{t}$ into (2.8), we find

$$
\begin{aligned}
0 & \leq t\left\langle A v_{t}-f, v-u\right\rangle_{X}+\varphi\left(u, v_{t}\right)-\varphi(u, u)+J^{0}\left(v_{t} ; v_{t}-u\right) \\
& \leq t\left\langle A v_{t}-f, v-u\right\rangle_{X}+t \varphi(u, v)-t \varphi(u, u)+t J^{0}\left(v_{t} ; v-u\right),
\end{aligned}
$$

where we have used the convexity of $v \mapsto \varphi(u, v)$ and the positive homogeneity of $v \mapsto$ $J^{0}(u ; v)$. Hence,

$$
\left\langle A v_{t}-f, v-u\right\rangle_{X}+\varphi(u, v)-\varphi(u, u)+J^{0}\left(v_{t} ; v-u\right) \geq 0 .
$$

Note that $A$ is pseudomonotone, so, it is demicontinuous, see e.g. [42, Theorem 3.69]. Passing to the upper limit as $t \rightarrow 0^{+}$in (2.10), it gives

$$
\begin{aligned}
& \langle A u-f, v-u\rangle_{X}+\varphi(u, v)-\varphi(u, u)+J^{0}(u ; v-u) \\
& \quad \geq \limsup _{t \rightarrow 0^{+}}\left\langle A v_{t}-f, v-u\right\rangle_{X}+\varphi(u, v)-\varphi(u, u)+\limsup _{t \rightarrow 0^{+}} J^{0}\left(v_{t} ; v-u\right) \\
& \quad \geq \limsup _{t \rightarrow 0^{+}}\left(\left\langle A v_{t}-f, v-u\right\rangle_{X}+\varphi(u, v)-\varphi(u, u)+J^{0}\left(v_{t} ; v-u\right)\right) \geq 0
\end{aligned}
$$

where we have applied Lemma 2.1(b). Recall that $v \in K$ is arbitrary, so, we conclude that $u \in K$ is a solution to Problem 2.1 as well. This completes the proof. 


\section{Main Results}

In this section, we are devoted to explore some global error estimates for variationalhemivariational inequality in Problem 2.1, by introducing concepts of a gap function, a regularized gap function, and the Moreau-Yosida regularized gap function associated to Problem 2.1.

Invoking the idea of Yamashita-Fukushima in [49], we now introduce the definitions of a gap function and a regularized gap function for Problem 2.1.

Definition 3.1 A real-valued function $\pi: K \rightarrow \mathbb{R}$ is said to be a gap function for Problem 2.1 , if it satisfies the following properties:

(a) $\pi(u) \geq 0$ for all $u \in K$.

(b) $u^{*} \in K$ is such that $\pi\left(u^{*}\right)=0$ if and only if $u^{*}$ is a solution to Problem 2.1.

Consider the functions $\Theta^{f}, \Theta_{*}^{f}: K \rightarrow \mathbb{R}$ defined by

$$
\begin{aligned}
& \Theta^{f}(u)=\sup _{v \in K}\left(\langle A u-f, u-v\rangle_{X}+\varphi(u, u)-\varphi(u, v)-J^{0}(u ; v-u)\right), \\
& \Theta_{*}^{f}(u)=\sup _{v \in K}\left(\langle A v-f, u-v\rangle_{X}+\varphi(u, u)-\varphi(u, v)-J^{0}(v ; v-u)\right)
\end{aligned}
$$

for all $u \in K$, respectively.

The following proposition shows that functions $\Theta^{f}$ and $\Theta_{*}^{f}$ are gap functions for Problem 2.1 .

Proposition 3.1 Suppose the assumptions of Theorem 2.1. Then, the functions $\Theta^{f}$ and $\Theta_{*}^{f}$ defined by (3.1) and (3.2) are two gap functions for Problem 2.1.

Proof In what follows, we prove that $\Theta^{f}$ is a gap function for Problem 2.1. In an analogous way, it is not difficult to show that the function $\Theta_{*}^{f}$ is also a gap function for Problem 2.1. We will verify two conditions of Definition 3.1.

(a) In fact, it is obvious that $\Theta^{f}(u) \geq 0$ for all $u \in K$. This property holds since for all $u \in K$, we have

$$
\Theta^{f}(u) \geq\langle A u-f, u-u\rangle_{X}+\varphi(u, u)-\varphi(u, u)-J^{0}(u ; u-u)=-J^{0}(u ; 0)=0 .
$$

(b) Suppose that $u^{*} \in K$ is such that $\Theta^{f}\left(u^{*}\right)=0$, i.e.,

$$
\sup _{v \in K}\left(\left\langle A u^{*}-f, u^{*}-v\right\rangle_{X}+\varphi\left(u^{*}, u^{*}\right)-\varphi\left(u^{*}, v\right)-J^{0}\left(u^{*} ; v-u^{*}\right)\right)=0 .
$$

This together with the fact

$$
\left\langle A u^{*}-f, u^{*}-u^{*}\right\rangle+\varphi\left(u^{*}, u^{*}\right)-\varphi\left(u^{*}, u^{*}\right)+J^{0}\left(u^{*} ; u^{*}-u^{*}\right)=0
$$

implies that (3.3) is equivalent to

$$
\left\langle A u^{*}-f, v-u^{*}\right\rangle_{X}+\varphi\left(u^{*}, v\right)-\varphi\left(u^{*}, u^{*}\right)+J^{0}\left(u^{*} ; v-u^{*}\right) \geq 0
$$

for all $v \in K$. Therefore, we conclude that $u^{*}$ is a solution to Problem 2.1 if and only if $\Theta^{f}\left(u^{*}\right)=0$. 
Let $\gamma>0$ be a fixed parameter. We consider the following functions $\Theta^{f, \gamma}, \Theta_{*}^{f, \gamma}: K \rightarrow \mathbb{R}$ defined by

$$
\begin{aligned}
& \Theta^{f, \gamma}(u)=\sup _{v \in K}\left(\langle A u-f, u-v\rangle_{X}+\varphi(u, u)-\varphi(u, v)-J^{0}(u ; v-u)-\frac{1}{2 \gamma}\|u-v\|_{X}^{2}\right), \\
& \Theta_{*}^{f, \gamma}(u)=\sup _{v \in K}\left(\langle A v-f, u-v\rangle_{X}+\varphi(u, u)-\varphi(u, v)-J^{0}(v ; v-u)-\frac{1}{2 \gamma}\|u-v\|_{X}^{2}\right)
\end{aligned}
$$

for all $u \in K$, respectively. In what follows, the functions $\Theta^{f, \gamma}$ and $\Theta_{*}^{f, \gamma}$ are called to be regularized gap functions for Problem 2.1.

We now assert that $\Theta^{f, \gamma}$ and $\Theta_{*}^{f, \gamma}$ are two gap functions of Problem 2.1.

Theorem 3.1 Suppose the hypotheses of Theorem 2.1. Then, for any $\gamma>0$, the functions $\Theta^{f, \gamma}$ and $\Theta_{*}^{f, \gamma}$ are gap functions for Problem 2.1 .

Proof We prove that $\Theta^{f, \gamma}$ is a gap function for Problem 2.1. Employing the analogous approach, it is not difficult to show that $\Theta_{*}^{f, \gamma}$ is also a gap function for Problem 2.1. We will check two conditions of Definition 3.1.

(a) For each $\gamma>0$ fixed, it is trivial that for each $u \in K$ it holds $\Theta^{f, \gamma}(u) \geq 0$. This is due to $u \in K$ and

$$
\begin{aligned}
\Theta^{f, \gamma}(u) & \geq\langle A u-f, u-u\rangle_{X}+\varphi(u, u)-\varphi(u, u)-J^{0}(u ; u-u)-\frac{1}{2 \gamma}\|u-u\|_{X}^{2} \\
& =-J^{0}(u ; 0)=0 .
\end{aligned}
$$

(b) Assume that $u^{*} \in K$ is such that $\Theta^{f, \gamma}\left(u^{*}\right)=0$, namely,

$$
\sup _{v \in K}\left(\left\langle A u^{*}-f, u^{*}-v\right\rangle_{X}+\varphi\left(u^{*}, u^{*}\right)-\varphi\left(u^{*}, v\right)-J^{0}\left(u^{*} ; v-u^{*}\right)-\frac{1}{2 \gamma}\left\|u^{*}-v\right\|_{X}^{2}\right)=0 .
$$

This means

$$
\left\langle A u^{*}-f, v-u^{*}\right\rangle_{X}+\varphi\left(u^{*}, v\right)-\varphi\left(u^{*}, u^{*}\right)+J^{0}\left(u^{*} ; v-u^{*}\right) \geq-\frac{1}{2 \gamma}\left\|u^{*}-v\right\|_{X}^{2}
$$

for all $v \in K$. For any $w \in K$ and $t \in(0,1)$, we insert $v=v_{t}:=(1-t) u^{*}+t w \in K$ into the above inequality to obtain

$$
\begin{aligned}
& t\left\langle A u^{*}-f, w-u^{*}\right\rangle_{X}+t \varphi\left(u^{*}, w\right)-t \varphi\left(u^{*}, u^{*}\right)+t J^{0}\left(u^{*} ; w-u^{*}\right) \\
& \quad \geq\left\langle A u^{*}-f, v_{t}-u^{*}\right\rangle_{X}+\varphi\left(u^{*}, v_{t}\right)-\varphi\left(u^{*}, u^{*}\right)+J^{0}\left(u^{*} ; v_{t}-u^{*}\right) \\
& \quad \geq-\frac{1}{2 \gamma}\left\|u^{*}-v_{t}\right\|_{X}^{2}=-\frac{t^{2}}{2 \gamma}\left\|u^{*}-w\right\|_{X}^{2},
\end{aligned}
$$

where we have used the convexity of $v \mapsto \varphi(u, v)$ and positive homogeneity of $v \mapsto$ $J^{0}(u ; v)$. Hence, we have

$$
\left\langle A u^{*}-f, w-u^{*}\right\rangle_{X}+\varphi\left(u^{*}, w\right)-\varphi\left(u^{*}, u^{*}\right)+J^{0}\left(u^{*} ; w-u^{*}\right) \geq-\frac{t}{2 \gamma}\left\|u^{*}-w\right\|_{X}^{2}
$$


for all $w \in K$. Letting $t \rightarrow 0^{+}$for the above inequality, it gives

$$
\left\langle A u^{*}-f, w-u^{*}\right\rangle_{X}+\varphi\left(u^{*}, w\right)-\varphi\left(u^{*}, u^{*}\right)+J^{0}\left(u^{*} ; w-u^{*}\right) \geq 0
$$

for all $w \in K$. Hence, $u^{*}$ is also a solution to Problem 2.1.

Conversely, suppose that $u^{*} \in K$ is a solution of Problem 2.1, that is,

$$
\left\langle A u^{*}-f, v-u^{*}\right\rangle_{X}+\varphi\left(u^{*}, v\right)-\varphi\left(u^{*}, u^{*}\right)+J^{0}\left(u^{*} ; v-u^{*}\right) \geq 0
$$

for all $v \in K$. This implies

$$
\sup _{v \in K}\left(\left\langle A u^{*}-f, u^{*}-v\right\rangle_{X}+\varphi\left(u^{*}, u^{*}\right)-\varphi\left(u^{*}, v\right)-J^{0}\left(u^{*} ; v-u^{*}\right)-\frac{1}{2 \gamma}\left\|u^{*}-v\right\|_{X}^{2}\right) \leq 0 .
$$

The latter combined with the fact $\Theta^{f, \gamma}(u) \geq 0$ for all $u \in K$ reveals that $\Theta^{f, \gamma}\left(u^{*}\right)=0$. This completes the proof.

Further, we will show that the regularized gap functions $\Theta^{f, \gamma}$ and $\Theta_{*}^{f, \gamma}$ are both lower semicontinuous.

Lemma 3.1 Assume that the hypotheses of Theorem 2.1 are satisfied. If, in addition, $\varphi: K \times$ $K \rightarrow \mathbb{R}$ is continuous, then, for each $\gamma>0$, the functions $\Theta^{f, \gamma}$ and $\Theta_{*}^{f, \gamma}$ are both lower semicontinuous.

Proof We will prove that $\Theta^{f, \gamma}$ is lower semicontinuous for each $\gamma>0$. It is not difficult to apply a similar argument to verify that $\Theta_{*}^{f, \gamma}$ has the same property.

Consider the function $\widehat{\Theta}^{f, \gamma}: K \times K \rightarrow \mathbb{R}$ defined by

$$
\widehat{\Theta}^{f, \gamma}(u, v)=\langle A u-f, u-v\rangle_{X}+\varphi(u, u)-\varphi(u, v)-J^{0}(u ; v-u)-\frac{1}{2 \gamma}\|u-v\|_{X}^{2} .
$$

Recall that the operator $A: X \rightarrow X^{*}$ is demicontinuous being pseudomonotone. This means that the function $u \mapsto\langle A u, u\rangle_{X}$ is continuous. The latter together with the lower semicontinuity of $(u, v) \mapsto-J^{0}(u ; v)$, and the continuity of $(u, v) \mapsto \varphi(u, v)$ and $u \mapsto\|u\|_{X}$ guarantees that $u \mapsto \widehat{\Theta}^{f, \gamma}(u, v)$ is lower semicontinuous for all $v \in K$.

Next, we observe that

$$
\Theta^{f, \gamma}(u)=\sup _{v \in K} \widehat{\Theta}^{f, \gamma}(u, v) \quad \text { for all } u \in K
$$

Let $\left\{u_{n}\right\} \subset K$ be such that $u_{n} \rightarrow u$ as $n \rightarrow \infty$. Then, we have

$$
\begin{aligned}
\liminf _{n \rightarrow \infty} \Theta^{f, \gamma}\left(u_{n}\right) & =\liminf _{n \rightarrow \infty} \sup _{v \in K} \widehat{\Theta}^{f, \gamma}\left(u_{n}, v\right) \\
& \geq \liminf _{n \rightarrow \infty} \widehat{\Theta}^{f, \gamma}\left(u_{n}, w\right) \geq \widehat{\Theta}^{f, \gamma}(u, w)
\end{aligned}
$$

for all $w \in K$. Passing to supremum with $w \in K$ for the above inequality, it gives

$$
\liminf _{n \rightarrow \infty} \Theta^{f, \gamma}\left(u_{n}\right) \geq \sup _{w \in K} \widehat{\Theta}^{f, \gamma}(u, w)=\Theta^{f, \gamma}(u),
$$

so, the function $\Theta^{f, \gamma}$ is lower semicontinuous. This completes the proof. 
Let $\gamma, \zeta>0$ be two parameters. Moreover, let us consider the following functions $\Pi_{\Theta}^{f, \gamma, \zeta}, \Pi_{\Theta_{*}^{f, \gamma, \zeta}}: K \rightarrow \mathbb{R}$ defined by

$$
\begin{aligned}
& \Pi_{\Theta f, \gamma, \zeta}(u)=\inf _{w \in K}\left\{\Theta^{f, \gamma}(w)+\zeta\|u-w\|_{X}^{2}\right\}, \\
& \Pi_{\Theta_{*}^{f, \gamma, \zeta}}(u)=\inf _{w \in K}\left\{\Theta_{*}^{f, \gamma}(w)+\zeta\|u-w\|_{X}^{2}\right\}
\end{aligned}
$$

for all $u \in K$, respectively. In the sequel, we call the functions $\Pi_{\Theta f, \gamma, \zeta}$ and $\Pi_{\Theta *}^{f, \gamma, \zeta}$ to be the Moreau-Yosida regularized gap functions for Problem 2.1. Subsequently, we will verify that these functions are two gap functions for Problem 2.1.

Theorem 3.2 Assume that the hypotheses of Lemma 3.1 are satisfied. Then, for all $\gamma, \zeta>0$, the functions $\Pi_{\Theta} f, \gamma, \zeta$ and $\Pi_{\Theta}{ }_{*}^{f, \gamma, \zeta}$ are two gap functions for Problem 2.1.

Proof We will show that $\Pi_{\Theta f, \gamma, \zeta}$ is a gap function for Problem 2.1. In an analogous way, it is possible to demonstrate that $\Pi_{\Theta_{*}^{f, \gamma, \zeta}}$ is also a gap function for Problem 2.1.

(a) For any $\gamma, \zeta>0$ fixed, recall that $\Theta^{f, \gamma}$ is a gap function for Problem 2.1, hence $\Theta^{f, \gamma}(u) \geq 0$ for all $u \in K$. In consequence, $\Pi_{\Theta f, \gamma, \zeta}(u) \geq 0$ for all $u \in K$.

(b) Suppose that $u^{*} \in K$ is a solution to Problem 2.1. Theorem 3.1 indicates that $\Theta^{f, \gamma}\left(u^{*}\right)=0$. Moreover, the inequality

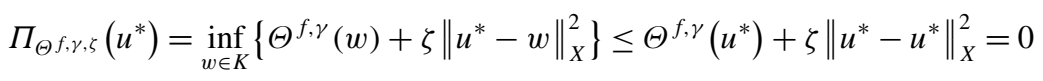

and the fact $\Pi_{\Theta f, \gamma, \zeta}\left(u^{*}\right) \geq 0$ imply that $\Pi_{\Theta f, \gamma, \zeta}\left(u^{*}\right)=0$.

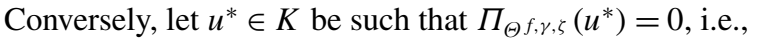

$$
\inf _{w \in K}\left\{\Theta^{f, \gamma}(w)+\zeta\left\|u^{*}-w\right\|_{X}^{2}\right\}=0 .
$$

Therefore, there exists a minimizing sequence $\left\{w_{n}\right\}$ in $K$ such that

$$
0 \leq \Theta^{f, \gamma}\left(w_{n}\right)+\zeta\left\|u^{*}-w_{n}\right\|_{X}^{2}<\frac{1}{n} .
$$

It is obvious that $\Theta^{f, \gamma}\left(w_{n}\right) \rightarrow 0$ and $\left\|u^{*}-w_{n}\right\|_{X} \rightarrow 0$, as $n \rightarrow \infty$. This implies $w_{n} \rightarrow u^{*}$, as $n \rightarrow+\infty$. Invoking Lemma 3.1 and nonnegativity of $\Theta^{f, \gamma}$ results in the inequality

$$
0 \leq \Theta^{f, \gamma}\left(u^{*}\right) \leq \liminf _{n \rightarrow+\infty} \Theta^{f, \gamma}\left(w_{n}\right)=0,
$$

thus is, $\Theta^{f, \gamma}\left(u^{*}\right)=0$. Because $\Theta^{f, \gamma}$ is a gap function, therefore, $u^{*}$ is a solution to Problem 2.1. The proof is complete.

We now provide an example to illustrate the results of Theorems 3.1 and 3.2.

Example 3.1 Let $X=\mathbb{R}, K=\left[\frac{1}{2}, \frac{3}{2}\right], f=1$ and $A: \mathbb{R} \rightarrow \mathbb{R}, \varphi: K \times K \rightarrow \mathbb{R}$ and $J: \mathbb{R} \rightarrow$ $\mathbb{R}$ be the functions defined by

$$
A(u)=5 u, \quad \varphi(u, v)=\frac{u v^{2}+1}{2}, \quad \text { and } \quad J(u)= \begin{cases}u^{2} & \text { if } u>0 \\ 2 u & \text { if } u \leq 0\end{cases}
$$


It is obvious that $J$ is a locally Lipschitz function and

$$
J^{0}(u ; d)= \begin{cases}2 u d & \text { if } u>0 \\ \max \{0,2 d\} & \text { if } u=0 \\ 2 d & \text { if } u<0\end{cases}
$$

for all $u, d \in X$. Besides, it is not difficult to verify that all assumptions of Theorem 2.1 are valid with $m_{A}=5, m_{J}=2$ and $\alpha_{\varphi}=\frac{3}{2}$. Using Theorem 2.1, we deduce that the following inequality has a unique solution $u=\frac{1}{2}$ : find $u \in K$ such that

$$
\begin{aligned}
& (5 u-1)(v-u)+\frac{u v^{2}+1}{2}-\frac{u^{3}+1}{2}+2 u(v-u) \\
& =\frac{1}{2}(v-u)\left(u^{2}+u v+14 u-2\right) \geq 0
\end{aligned}
$$

for all $v \in K$. Next, let $\gamma=1$. For the problem (3.9), we consider the regularized function $\Theta^{f, \gamma}$ defined in (3.4). A simple calculation gives

$$
\begin{aligned}
\Theta^{f, \gamma}(u) & =\sup _{v \in K}\left(\langle A u-f, u-v\rangle_{X}+\varphi(u, u)-\varphi(u, v)-J^{0}(u ; v-u)-\frac{1}{2 \gamma}\|u-v\|_{X}^{2}\right) \\
& =\sup _{v \in\left[\frac{1}{2}, \frac{3}{2}\right]}\left(\frac{1}{2}(u-v)\left(u^{2}+u v+14 u-2\right)-\frac{1}{2}(u-v)^{2}\right) \\
& =\max _{v \in\left[\frac{1}{2}, \frac{3}{2}\right]}\left(\frac{1}{2}(u-v)\left(u^{2}+13 u-2+(u+1) v\right)\right) \\
& =\frac{1}{2}\left(u-\frac{1}{2}\right)\left(2 u^{2}+27 u-3\right) .
\end{aligned}
$$

Observe that $\Theta^{f, \gamma}(u) \geq 0$ for all $u \in K$, and $\Theta^{f, \gamma}(u)=0$ if and only if $u=\frac{1}{2}$. This means that $\Theta^{f, \gamma}$ is a gap function for the problem (3.9).

Let $\gamma=1$ and $\zeta=\frac{1}{2}$. The Moreau-Yosida regularized gap function for the problem (3.9) can be calculated as follows

$$
\begin{aligned}
\Pi_{\Theta f, \gamma, \zeta}(u) & =\inf _{w \in K}\left\{\Theta^{f, \gamma}(w)+\zeta\|u-w\|_{X}^{2}\right\} \\
& =\inf _{w \in\left[\frac{1}{2}, \frac{3}{2}\right]}\left\{\frac{1}{4}\left(w-\frac{1}{2}\right)\left(2 w^{2}+27 w-3\right)+\frac{1}{2}(u-w)^{2}\right\} \\
& =\min _{w \in\left[\frac{1}{2}, \frac{3}{2}\right]}\left\{\frac{w^{3}}{2}+7 w^{2}-\left(u+\frac{33}{8}\right) w+\frac{u^{2}}{2}+\frac{3}{8}\right\} \\
& =\frac{1}{4}\left(u-\frac{1}{2}\right)^{2} .
\end{aligned}
$$

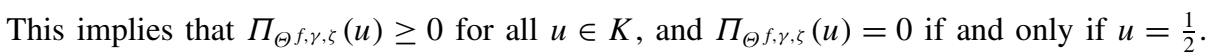
Therefore, $\Pi_{\Theta f, \gamma, \zeta}$ is also a gap function for the problem (3.9).

Analogously, it also can proved that the regularized gap function $\Theta_{*}^{f, \gamma}$ and the MoreauYosida regularized gap function $\Pi_{\Theta_{*}^{f, \gamma, \zeta}}$ are two gap functions for the problem (3.9). 
We conclude this section with two global error bounds for Problem 2.1 associated with the regularized gap function $\Theta^{f, \gamma}$ and the Moreau-Yosida regularized gap function $\Pi_{\Theta f, \gamma, \zeta}$, respectively. These global error estimates measure the distance between any admissible point and the unique solution to Problem 2.1.

Theorem 3.3 Let $u^{*} \in K$ be the unique solution to Problem 2.1 and $\gamma>0$ be such that $m_{A}-\alpha_{\varphi}-m_{J}>\frac{1}{2 \gamma}$. Assume that the hypotheses of Theorem 2.1 hold. Then, for each $u \in K$, we have

$$
\left\|u-u^{*}\right\|_{X} \leq \sqrt{\frac{\Theta^{f, \gamma}(u)}{m_{A}-\alpha_{\varphi}-m_{J}-\frac{1}{2 \gamma}}} .
$$

Proof Let $u^{*} \in K$ be the unique solution to Problem 2.1, i.e.,

$$
\left\langle A u^{*}-f, v-u^{*}\right\rangle_{X}+\varphi\left(u^{*}, v\right)-\varphi\left(u^{*}, u^{*}\right)+J^{0}\left(u^{*} ; v-u^{*}\right) \geq 0
$$

for all $v \in K$. For any $u \in K$ fixed, we insert $v=u$ into the above inequality to obtain

$$
\left\langle A u^{*}-f, u-u^{*}\right\rangle_{X}+\varphi\left(u^{*}, u\right)-\varphi\left(u^{*}, u^{*}\right)+J^{0}\left(u^{*} ; u-u^{*}\right) \geq 0 .
$$

By virtue of the definition of $\Theta^{f, \gamma}$, one has

$$
\Theta^{f, \gamma}(u) \geq\left\langle A u-f, u-u^{*}\right\rangle_{X}+\varphi(u, u)-\varphi\left(u, u^{*}\right)-J^{0}\left(u ; u^{*}-u\right)-\frac{1}{2 \gamma}\left\|u-u^{*}\right\|_{X}^{2} .
$$

It follows from the monotonicity of $A$, hypotheses (2.2)(b) and (2.3)(b) that

$$
\begin{aligned}
\left\langle A u-f, u-u^{*}\right\rangle_{X}+\varphi(u, u)-\varphi\left(u, u^{*}\right)-J^{0}\left(u ; u^{*}-u\right)-\frac{1}{2 \gamma}\left\|u-u^{*}\right\|_{X}^{2} \\
\geq\left\langle A u^{*}-f, u-u^{*}\right\rangle_{X}+\varphi\left(u^{*}, u\right)-\varphi\left(u^{*}, u^{*}\right)+J^{0}\left(u^{*} ; u-u^{*}\right) \\
\quad+\left(m_{A}-m_{J}-\alpha_{\varphi}-\frac{1}{2 \gamma}\right)\left\|u-u^{*}\right\|_{X}^{2} \\
\geq\left(m_{A}-m_{J}-\alpha_{\varphi}-\frac{1}{2 \gamma}\right)\left\|u-u^{*}\right\|_{X}^{2},
\end{aligned}
$$

where the last inequality is obtained by using (3.11). Combining the last two inequality, we have

$$
\Theta^{f, \gamma}(u) \geq\left(m_{A}-\alpha_{\varphi}-m_{J}-\frac{1}{2 \gamma}\right)\left\|u-u^{*}\right\|_{X}^{2} .
$$

Hence, the desired inequality (3.10) is valid.

Theorem 3.4 Let $u^{*} \in K$ be the unique solution to Problem 2.1 and $\gamma>0$ be such that $m_{A}-\alpha_{\varphi}-m_{J}>\frac{1}{2 \gamma}$. Assume that the hypotheses of Theorem 2.1 hold. Then, for each $u \in K$ and all $\zeta>0$, we have

$$
\left\|u-u^{*}\right\|_{X} \leq \sqrt{\frac{2 \Pi_{\Theta} f, \gamma, \zeta}{\min \left\{m_{A}-\alpha_{\varphi}-m_{J}-\frac{1}{2 \gamma}, \zeta\right\}}} .
$$

Proof Let $u^{*} \in K$ be the unique solution of Problem 2.1. By the definition of the function $\Pi_{\Theta} f, \gamma, \zeta$, it follows

$$
\Pi_{\Theta f, \gamma, \zeta}(u)=\inf _{w \in K}\left\{\Theta^{f, \gamma}(w)+\zeta\|u-w\|_{X}^{2}\right\}
$$




$$
\begin{aligned}
& \geq \inf _{w \in K}\left\{\left(m_{A}-\alpha_{\varphi}-m_{J}-\frac{1}{2 \gamma}\right)\left\|u^{*}-w\right\|_{X}^{2}+\zeta\|u-w\|_{X}^{2}\right\} \\
& \geq \min \left\{m_{A}-\alpha_{\varphi}-m_{J}-\frac{1}{2 \gamma}, \zeta\right\} \inf _{w \in K}\left\{\left\|u^{*}-w\right\|_{X}^{2}+\|u-w\|_{X}^{2}\right\} \\
& \geq \frac{1}{2} \min \left\{m_{A}-\alpha_{\varphi}-m_{J}-\frac{1}{2 \gamma}, \zeta\right\}\left\|u-u^{*}\right\|_{X}^{2}
\end{aligned}
$$

for all $u \in K$. Hence

$$
\left\|u-u^{*}\right\|_{X} \leq \sqrt{\frac{2 \Pi_{\Theta f, \gamma, \zeta}(u)}{\min \left\{m_{A}-\alpha_{\varphi}-m_{J}-\frac{1}{2 \gamma}, \zeta\right\}}}
$$

for all $u \in K$, which completes the proof of the theorem.

Finally, we will illustrate the results of Theorems 3.3 and 3.4 by the following example.

Example 3.2 Under the framework of Example 3.1, for the regularized gap function $\Theta^{f, \gamma}$ and the Moreau-Yosida regularized gap function $\Pi_{\Theta} f, \gamma, \zeta$, it is easy to compute the global error bounds for the problem (3.9):

$$
\begin{aligned}
\sqrt{\frac{\Theta^{f, \gamma}(u)}{m_{A}-\alpha_{\varphi}-m_{J}-\frac{1}{2 \gamma}}} & =\sqrt{\frac{1}{4}\left(u-\frac{1}{2}\right)\left(2 u^{2}+27 u-3\right)} \\
& \geq \sqrt{\frac{42}{4}\left(u-\frac{1}{2}\right)^{2}} \geq\left|u-\frac{1}{2}\right|
\end{aligned}
$$

and

$$
\sqrt{\frac{2 \Pi_{\Theta f, \gamma, \zeta}(u)}{\min \left\{m_{A}-\alpha_{\varphi}-m_{J}-\frac{1}{2 \gamma}, \zeta\right\}}}=\sqrt{\frac{\left(u-\frac{1}{2}\right)^{2}}{\min \left\{1, \frac{1}{2}\right\}}} \geq\left|u-\frac{1}{2}\right| .
$$

\section{Application to an Elliptic Boundary Value Problem}

In the section we shall investigate a boundary value problem with the generalized gradient and an obstacle effect which illustrates the applicability of the abstract results.

Let $\Omega$ be a bounded domain in $\mathbb{R}^{d}(d=2,3)$ with Lipschitz continuous boundary $\Gamma$. The boundary is divided into two mutually disjoint measurable parts $\Gamma_{1}$ and $\Gamma_{2}$ such that meas $\left(\Gamma_{1}\right)>0$. Consider the following elliptic mixed boundary value problem with constraints.

Problem 4.1 Find a function $u: \Omega \rightarrow \mathbb{R}$ such that

$$
\begin{array}{ll}
-\operatorname{div} a(\boldsymbol{x}, \nabla u)+\partial g(\boldsymbol{x}, u) \ni f(\boldsymbol{x}) & \text { in } \Omega, \\
u(\boldsymbol{x}) \leq \Phi(\boldsymbol{x}) & \text { in } \Omega, \\
u=0 & \text { on } \Gamma_{1}, \\
-\frac{\partial u}{\partial \nu_{a}} \in k(u) \partial_{c} h(\boldsymbol{x}, u) & \text { on } \Gamma_{2} .
\end{array}
$$


Here $\partial g$ and $\partial_{c} h$ denote the generalized gradient and the convex subdifferential of the functions $g: \Omega \times \mathbb{R} \rightarrow \mathbb{R}$ and $h: \Gamma_{2} \times \mathbb{R} \rightarrow \mathbb{R}$ respectively with respect to their second variables, while the conormal derivative $\frac{\partial u}{\partial v_{a}}=(a(\boldsymbol{x}, \nabla u), \boldsymbol{v})_{\mathbb{R}^{d}}$ represents the heat flux through the part $\Gamma_{2}$, where $v$ stands for the outward unit normal on $\Gamma$.

The mathematical model (4.1)-(4.4) is motivated by the study of semipermeability phenomena which may appear in the interior and on the boundary of the body $\Omega$, and are met, for instance, in electrostatics, magnetostatics or stationary heat transfer (the behavior of natural and artificial semipermeable membranes of finite thickness, temperature control problems, etc.), see [44-46, 50] and the references therein. The function $u$ represents the electric potential, magnetic potential or temperature, respectively, the function $a=a(\boldsymbol{x}, \nabla u)$ is the dielectric coefficient, magnetic permeability or thermal conductivity, and $f=f(\boldsymbol{x})$ is a given source term. The material which occupies $\Omega$ is non-isotropic and heterogeneous, and thus $a$ effectively depends on $\boldsymbol{x}$. Condition (4.2) represents an additional unilateral constraint for the solution. Since the function $g(\boldsymbol{x}, \cdot)$ is supposed to be locally Lipschitz for a.e. $\boldsymbol{x} \in \Omega$, but not necessary convex, the multivalued relation (4.1) is nonmonotone in general. Combining it with (4.2)-(4.4) leads to a variational formulation which is a constrained variational-hemivariational inequality. Note that in general there is no function $\widetilde{h}$ such that $\partial \widetilde{h}=k \partial_{c} h$. This means that if $g \equiv 0$, then the weak form of Problem 4.1, stated in Problem 4.2 below, reduces to quasi-variational inequality.

We need the following standard functional space. Let $X$ be defined by

$$
X=\left\{v \in H^{1}(\Omega) \mid v=0 \text { on } \Gamma_{1}\right\} .
$$

Since meas $\left(\Gamma_{1}\right)>0$, the space $X$ is endowed with the inner product and corresponding norm given by

$$
\langle u, v\rangle_{X}=\int_{\Omega}(\nabla u(\boldsymbol{x}), \nabla v(\boldsymbol{x}))_{\mathbb{R}^{d}} d \boldsymbol{x} \quad \text { and } \quad\|v\|_{X}:=\left(\int_{\Omega}\|\nabla v(\boldsymbol{x})\|_{\mathbb{R}^{d}}^{2} d \boldsymbol{x}\right)^{\frac{1}{2}}
$$

for all $u, v \in X$. Also, we denote by $\gamma_{0}: X \rightarrow L^{2}(\Gamma)$ the trace operator. On the other hand, we consider the admissible set $K$ defined by

$$
K:=\{v \in X \mid v(\boldsymbol{x}) \leq \Phi(\boldsymbol{x}) \text { for a.e. } \boldsymbol{x} \in \Omega\}
$$

In order to provide the result on the unique solvability of Problem 4.1, we need the following hypotheses on the data.

$$
\left\{\begin{array}{l}
a: \Omega \times \mathbb{R}^{d} \rightarrow \mathbb{R}^{d} \text { is such that } \\
\text { (a) } a(\cdot, \boldsymbol{z}) \text { is measurable on } \Omega \text { for all } z \in \mathbb{R}^{d} \\
\text { with } a(\boldsymbol{x}, \mathbf{0})=\mathbf{0} \text { for a.e. } \boldsymbol{x} \in \Omega \text {. } \\
\text { (b) } a(\boldsymbol{x}, \cdot) \text { is continuous on } \mathbb{R}^{d} \text { for a.e. } \boldsymbol{x} \in \Omega \text {. } \\
\text { (c) }\|a(\boldsymbol{x}, \boldsymbol{z})\|_{\mathbb{R}^{d}} \leq m_{a}\left(1+\|z\|_{\mathbb{R}^{d}}\right) \text { for all } z \in \mathbb{R}^{d}, \text { a.e. } \boldsymbol{x} \in \Omega \text { with } m_{a}>0 . \\
\text { (d) }\left(a\left(\boldsymbol{x}, \boldsymbol{z}_{1}\right)-a\left(\boldsymbol{x}, \boldsymbol{z}_{2}\right)\right) \cdot\left(\boldsymbol{z}_{1}-\boldsymbol{z}_{2}\right) \geq \alpha_{a}\left\|z_{1}-z_{2}\right\|_{\mathbb{R}^{d}}^{2} \\
\text { for all } \boldsymbol{z}_{1}, \boldsymbol{z}_{2} \in \mathbb{R}^{d} \text { and a.e. } \boldsymbol{x} \in \Omega \text { with } \alpha_{a}>0 .
\end{array}\right.
$$


$g: \Omega \times \mathbb{R} \rightarrow \mathbb{R}$ is such that

(a) $g(\cdot, r)$ is measurable on $\Omega$ for all $r \in \mathbb{R}$ and there exists $\tilde{e} \in L^{2}(\Omega)$ such that $g(\cdot, \widetilde{e}(\cdot)) \in L^{1}(\Omega)$.

(b) $g(\boldsymbol{x}, \cdot)$ is locally Lipschitz on $\mathbb{R}$ for a.e. $\boldsymbol{x} \in \Omega$.

(c) there exist $\bar{c}_{0}, \bar{c}_{1} \geq 0$ such that

$|\partial g(\boldsymbol{x}, r)| \leq \bar{c}_{0}+\bar{c}_{1}|r|$ for all $r \in \mathbb{R}$ and a.e. $\boldsymbol{x} \in \Omega$.

(d) there exists $\alpha_{g} \geq 0$ such that

$$
g^{0}\left(\boldsymbol{x}, r_{1} ; r_{2}-r_{1}\right)+g^{0}\left(\boldsymbol{x}, r_{2} ; r_{1}-r_{2}\right) \leq \alpha_{g}\left|r_{1}-r_{2}\right|^{2}
$$

for all $r_{1}, r_{2} \in \mathbb{R}$ and a.e. $\boldsymbol{x} \in \Omega$.

$h: \Gamma_{2} \times \mathbb{R} \rightarrow \mathbb{R}$ is such that

(a) $h(\cdot, r)$ is measurable on $\Gamma_{2}$ for all $r \in \mathbb{R}$.

(b) $h(\boldsymbol{x}, \cdot)$ is convex on $\mathbb{R}$ for a.e. $\boldsymbol{x} \in \Omega$.

(c) there exists $L_{h}>0$ such that

$$
\left|h\left(\boldsymbol{x}, r_{1}\right)-h\left(\boldsymbol{x}, r_{2}\right)\right| \leq L_{h}\left|r_{1}-r_{2}\right|
$$

for all $r_{1}, r_{2} \in \mathbb{R}$ and a.e. $\boldsymbol{x} \in \Gamma_{2}$.

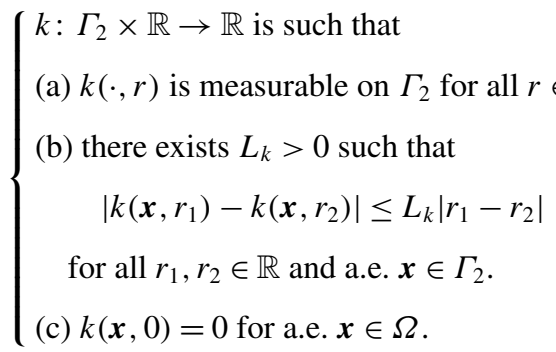

$$
\Phi \in X \quad \text { and } \quad f \in L^{2}(\Omega) .
$$

Moreover, using the standard technique based on the Green formula, see [42, 48, 50], we obtain the following variational formulation of Problem 4.1.

Problem 4.2 Find $u \in K$ such that

$$
\begin{aligned}
& \int_{\Omega}(a(\boldsymbol{x}, \nabla u), \nabla(v-u))_{\mathbb{R}^{d}} d \boldsymbol{x}+\int_{\Gamma_{2}}(k(u) h(\boldsymbol{x}, v)-k(u) h(\boldsymbol{x}, u)) d \Gamma \\
& \quad+\int_{\Omega} g^{0}(\boldsymbol{x}, u ; v-u) d \boldsymbol{x} \geq \int_{\Omega} f(v-u) d \boldsymbol{x} \quad \text { for all } v \in K .
\end{aligned}
$$

Theorem 4.1 Assume the hypotheses (4.5)-(4.9). If, in addition, the inequality holds

$$
\alpha_{a}-\alpha_{g}-L_{h} L_{k}\left\|\gamma_{0}\right\|^{2}>0,
$$

then Problem 4.2 has a unique solution $u^{*} \in K$. 
Proof Consider the operator $A: X \rightarrow X^{*}$ and the functions $\varphi: K \times K \rightarrow \mathbb{R}$ and $J: X \rightarrow \mathbb{R}$ defined by

$$
\begin{aligned}
\langle A u, v\rangle_{X} & =\int_{\Omega}(a(\boldsymbol{x}, \nabla u), \nabla v)_{\mathbb{R}^{d}} d \boldsymbol{x}, \\
\varphi(u, v) & =\int_{\Gamma_{2}} k(u) h(v) d \Gamma \\
J(v) & =\int_{\Omega} g(\boldsymbol{x}, v) d \boldsymbol{x}
\end{aligned}
$$

for all $u, v \in X$. It is easy to prove that all conditions of Theorem 2.1 are satisfied with $m_{A}=\alpha_{a}, m_{J}=\alpha_{g}, c_{0}=\bar{c}_{0}, c_{1}=\bar{c}_{1}$ and $\alpha_{\varphi}=L_{h} L_{k}\left\|\gamma_{0}\right\|^{2}$. Using Theorem 2.1 and the fact

$$
J^{0}(u ; v) \leq \int_{\Omega} g^{0}(u ; v) d \boldsymbol{x} \quad \text { for all } u, v \in X,
$$

we can conclude that Problem 4.2 admits a solution. Moreover, the smallness condition (4.10) guarantees that Problem 4.2 is uniquely solvable.

Next, for any parameter $\gamma>0$, we introduce the function $\widetilde{\Theta}^{f, \gamma}: K \rightarrow \mathbb{R}$ defined by

$$
\begin{aligned}
\widetilde{\Theta}^{f, \gamma}(u)=\sup _{v \in K} & \left(\int_{\Omega} a(\boldsymbol{x}, \nabla u) \cdot \nabla(u-v) d \boldsymbol{x}+\int_{\Gamma_{2}}(k(u) h(\boldsymbol{x}, u)-k(u) h(\boldsymbol{x}, v)) d \Gamma\right. \\
& \left.-\int_{\Omega} f(u-v) d \boldsymbol{x}-\int_{\Omega} g^{0}(\boldsymbol{x}, u ; v-u) d \boldsymbol{x}-\frac{1}{2 \gamma}\|u-v\|_{X}^{2}\right) .
\end{aligned}
$$

From Theorems 3.1-3.4 and 4.1, we directly obtain the following error estimates.

Theorem 4.2 Let $u^{*} \in K$ be the unique solution to Problem 4.2. Under the hypotheses of Theorem 4.1, we have

(i) for each $\gamma>0$ and $f \in L^{2}(\Omega), \widetilde{\Theta}^{f, \gamma}: K \rightarrow \mathbb{R}$ is a regularized gap function for Problem 4.2 .

(ii) if $\gamma>0$ is such that

$$
\alpha_{a}-\alpha_{g}-L_{h} L_{k}\left\|\gamma_{0}\right\|^{2}>\frac{1}{2 \gamma},
$$

then, for each $u \in K$, it holds

$$
\left\|u-u^{*}\right\|_{X} \leq \sqrt{\frac{\widetilde{\Theta}^{f, \gamma}(u)}{\alpha_{a}-\alpha_{p}-L_{h} L_{k}\left\|\gamma_{0}\right\|^{2}-\frac{1}{2 \gamma}}} .
$$

Theorem 4.3 Let $u^{*} \in K$ be the unique solution to Problem 4.2. Under the hypotheses of Theorem 4.1, we have

(i) for any $\gamma, \zeta>0$, the function $\widetilde{\Pi}_{\widetilde{\Theta} f, \gamma, \zeta}: K \rightarrow \mathbb{R}$ defined by

$$
\widetilde{\Pi}_{\widetilde{\Theta}, \gamma, \zeta}(u)=\inf _{w \in K}\left\{\widetilde{\Theta}^{f, \gamma}(w)+\zeta\|u-w\|_{X}^{2}\right\}
$$

is the Moreau-Yosida regularized gap function for Problem 4.2. 
(ii) for any $\zeta>0$, if $\gamma>0$ is such that

$$
\alpha_{a}-\alpha_{g}-L_{h} L_{k}\left\|\gamma_{0}\right\|^{2}>\frac{1}{2 \gamma}
$$

then, for each $u \in K$ the following bounds holds

$$
\left\|u-u^{*}\right\|_{X} \leq \sqrt{\frac{2 \widetilde{\Pi}_{\widetilde{\Theta}^{f, \gamma, \zeta}}(u)}{\min \left\{\alpha_{a}-\alpha_{g}-L_{h} L_{k}\left\|\gamma_{0}\right\|^{2}-\frac{1}{2 \gamma}, \zeta\right\}}} .
$$

Acknowledgements The authors wish to thank the anonymous referees for the careful reports and valuable comments that helped significantly improve the presentation of the paper. Project supported by the European Union's Horizon 2020 Research and Innovation Programme under the Marie Skłodowska-Curie grant agreement No. 823731 CONMECH, the National Science Center of Poland under Preludium Project No. 2017/25/N/ST1/00611, the NSF of Guangxi (Grant No: 2018GXNSFAA281353), the Ministry of Science and Higher Education of Republic of Poland under Grants Nos. 4004/GGPJII/H2020/2018/0 and 440328/PnH2/2019, and the Beibu Gulf University under Project No. 2018KYQD06.

Publisher's Note Springer Nature remains neutral with regard to jurisdictional claims in published maps and institutional affiliations.

Open Access This article is licensed under a Creative Commons Attribution 4.0 International License, which permits use, sharing, adaptation, distribution and reproduction in any medium or format, as long as you give appropriate credit to the original author(s) and the source, provide a link to the Creative Commons licence, and indicate if changes were made. The images or other third party material in this article are included in the article's Creative Commons licence, unless indicated otherwise in a credit line to the material. If material is not included in the article's Creative Commons licence and your intended use is not permitted by statutory regulation or exceeds the permitted use, you will need to obtain permission directly from the copyright holder. To view a copy of this licence, visit http://creativecommons.org/licenses/by/4.0/.

\section{References}

1. Anh, L.Q., Hung, N.V., Tam, V.M.: Regularized gap functions and error bounds for generalized mixed strong vector quasi equilibrium problems. Comput. Appl. Math. 37, 5935-5950 (2018)

2. Anh, L.Q., Bantaojai, T., Hung, N.V., Tam, V.M., Wangkeeree, R.: Painlevé-Kuratowski convergences of the solution sets for generalized vector quasi-equilibrium problems. Comput. Appl. Math. 37, 38323845 (2018)

3. Anh, L.Q., Hung, N.V.: Gap functions and Hausdorff continuity of solution mappings to parametric strong vector quasiequilibrium problems. J. Ind. Manag. Optim. 14, 65-79 (2018)

4. Auslender, A.: Optimisation: Méthodes Numériques. Masson, Paris (1976)

5. Aussel, D., Dutta, J.: On gap functions for multivalued Stampacchia variational inequalities. J. Optim. Theory Appl. 149, 513-527 (2011)

6. Aussel, D., Guptab, R., Mehrab, A.: Gap functions and error bounds for inverse quasi-variational inequality problems. J. Math. Anal. Appl. 407, 270-280 (2013)

7. Bigi, G., Passacantando, M.: $D$-gap functions and descent techniques for solving equilibrium problems. J. Glob. Optim. 62, 183-203 (2015)

8. Clarke, F.H.: Optimization and Nonsmooth Analysis. Wiley, New York (1983)

9. Denkowski, Z., Migórski, S., Papageorgiou, N.S.: An Introduction to Nonlinear Analysis: Theory. Kluwer Academic/Plenum, Dordrecht/New York (2003)

10. Denkowski, Z., Migórski, S., Papageorgiou, N.S.: An Introduction to Nonlinear Analysis: Applications. Kluwer Academic/Plenum, Dordrecht/New York (2003)

11. Fan, J.H., Wang, X.G.: Gap functions and global error bounds for set-valued variational inequalities. J. Comput. Appl. Math. 233, 2956-2965 (2010)

12. Fukushima, M.: Equivalent differentiable optimization problems and descent methods for asymmetric variational inequality problems. Math. Program. 53, 99-110 (1992) 
13. Fukushima, M.: A class of gap functions for quasi-variational inequality problems. J. Ind. Manag. Optim. 3, 165-171 (2007)

14. Gupta, R., Mehra, A.: Gap functions and error bounds for quasivariational inequalities. J. Glob. Optim. 53, 737-748 (2012)

15. Han, W.: Numerical analysis of stationary variational-hemivariational inequalities with applications in contact mechanics. Math. Mech. Solids 23, 279-293 (2018)

16. Han, W., Migórski, S., Sofonea, M.: A class of variational-hemivariational inequalities with applications to frictional contact problems. SIAM J. Math. Anal. 46, 3891-3912 (2014)

17. Han, W., Sofonea, M., Danan, D.: Numerical analysis of stationary variational-hemivariational inequalities. Numer. Math. 139, 563-592 (2018)

18. Han, W., Zeng, S.D.: On convergence of numerical methods for variational-hemivariational inequalities under minimal solution regularity. Appl. Math. Lett. 93, 105-110 (2019)

19. Hearn, D.W.: The gap function of a convex program. Oper. Res. Lett. 1, 67-71 (1982)

20. Hung, N.V.: Stability of a solution set for parametric generalized vector mixed quasi-variational inequality problem. J. Inequal. Appl. 2013, 276 (2013)

21. Hung, N.V.: On the lower semicontinuity of the solution sets for parametric generalized vector mixed quasivariational inequality problems. Bull. Korean Math. Soc. 52, 1777-1795 (2015)

22. Hung, N.V.: On the stability of the solution mapping for parametric traffic network problems. Indag. Math. 29, 885-894 (2018)

23. Hung, N.V., Tam, V.M., Elisabeth, K., Yao, J.C.: Existence of solutions and algorithm for generalized vector quasi-complementarity problems with application to traffic network problems. J. Nonlinear Convex Anal. 20, 1751-1775 (2019)

24. Hung, N.V., Tam, V.M., Tuan, N.H., O’Regan, D.: Regularized gap functions and error bounds for generalized mixed weak vector quasi variational inequality problems in fuzzy environments. Fuzzy Sets Syst. (2019). https://doi.org/10.1016/j.fss.2019.09.015

25. Hung, N.V., Tam, V.M., Pitea, A.: Global error bounds for mixed quasi-hemivariational inequality problems on Hadamard manifolds. Optimization (2020). https://doi.org/10.1080/02331934.2020.1718126

26. Hung, N.V., Tam, V.M., Baleanu, D.: Regularized gap functions and error bounds for split mixed vector quasivariational inequality problems. Math. Methods Appl. Sci. (2020). https://doi.org/10.1002/mma. 6219

27. Hung, N.V., Tam, V.M., O'Regan, D., Cho, Y.J.: A new class of generalized multiobjective games in bounded rationality with fuzzy mappings: structural $(\lambda, \varepsilon)$-stability and $(\lambda, \varepsilon)$-robustness to $\varepsilon$-equilibria. J. Comput. Appl. Math. 372, 112735 (2020)

28. Khan, S.A., Chen, J.W.: Gap function and global error bounds for generalized mixed quasi variational inequalities. Appl. Math. Comput. 260, 71-81 (2015)

29. Khan, S.A., Chen, J.W.: Gap functions and error bounds for generalized mixed vector equilibrium problems. J. Optim. Theory Appl. 166, 767-776 (2015)

30. Li, X., Liu, Z.H.: Sensitivity analysis of optimal control problems described by differential hemivariational inequalities. SIAM J. Control Optim. 56, 3569-3597 (2018)

31. Liu, Z.H., Motreanu, D.: Inclusion problems via subsolution-supersolution method with applications to hemivariational inequalities. Appl. Anal. 97, 1454-1465 (2018)

32. Liu, Z., Zeng, S.D., Zeng, B.: Well-posedness for mixed quasi-variational-hemivariational inequalities. Topol. Methods Nonlinear Anal. 47, 561-578 (2016)

33. Liu, Z.H., Zeng, S.D.: Differential variational inequalities in infinite Banach spaces. Acta Math. Sci. 37, 26-32 (2017)

34. Liu, Z.H., Zeng, S.D., Motreanu, D.: Evolutionary problems driven by variational inequalities. J. Differ. Equ. 260, 6787-6799 (2016)

35. Liu, Z.H., Zeng, S.D., Motreanu, D.: Partial differential hemivariational inequalities. Adv. Nonlinear Anal. 7, 571-586 (2018)

36. Liu, Z.H., Migórski, S., Zeng, S.D.: Partial differential variational inequalities involving nonlocal boundary conditions in Banach spaces. J. Differ. Equ. 263, 3989-4006 (2017)

37. Migórski, S., Zeng, S.D.: Hyperbolic hemivariational inequalities controlled by evolution equations with application to adhesive contact model. Nonlinear Anal., Real World Appl. 43, 121-143 (2018)

38. Migórski, S., Zeng, S.D.: A class of differential hemivariational inequalities in Banach spaces. J. Glob. Optim. 72, 761-779 (2018)

39. Migórski, S., Zeng, S.D.: A class of generalized evolutionary problems driven by variational inequalities and fractional operators. Set-Valued Var. Anal. 27, 949-970 (2019)

40. Migórski, S., Zeng, S.D.: Mixed variational inequalities driven by fractional evolution equations. Acta Math. Sci. 39, 461-468 (2019)

41. Migórski, S., Khan, A.A., Zeng, S.D.: Inverse problems for nonlinear quasi-hemivariational inequalities with application to mixed boundary value problems. Inverse Problems 36, ID: 024008 (2020). 20 pages 
42. Migórski, S., Ochal, A., Sofonea, M.: Nonlinear Inclusions and Hemivariational Inequalities. Models and Analysis of Contact Problems. Advances in Mechanics and Mathematics, vol. 26. Springer, New York (2013)

43. Migórski, S., Ochal, A., Sofonea, M.: A class of variational-hemivariational inequalities in reflexive Banach spaces. J. Elast. 127, 151-178 (2017)

44. Naniewicz, Z., Panagiotopoulos, P.D.: Mathematical Theory of Hemivariational Inequalities and Applications. Marcel Dekker, New York (1995)

45. Panagiotopoulos, P.D.: Nonconvex problems of semipermeable media and related topics. Z. Angew. Math. Mech. 65, 29-36 (1985)

46. Panagiotopoulos, P.D.: Hemivariational Inequalities, Applications in Mechanics and Engineering. Springer, Berlin (1993)

47. Sofonea, M., Migórski, S.: A class of history-dependent variational-hemivariational inequalities. Nonlinear Differ. Equ. Appl. 23, 38 (2016). https://doi.org/10.1007/s00030-016-0391-0

48. Sofonea, M., Migórski, S.: Variational-Hemivariational Inequalities with Applications. Pure and Applied Mathematics. Chapman \& Hall/CRC Press, Boca Raton/London (2018)

49. Yamashita, N., Fukushima, M.: Equivalent unconstrained minimization and global error bounds for variational inequality problems. SIAM J. Control Optim. 35, 273-284 (1997)

50. Zeng, B., Liu, Z., Migórski, S.: On convergence of solutions to variational-hemivariational inequalities. Z. Angew. Math. Phys. 69, 87 (2018). https://doi.org/10.1007/s00033-018-0980-3

51. Zeng, S.D., Liu, Z.H., Migórski, S.: A class of fractional differential hemivariational inequalities with application to contact problem. Z. Angew. Math. Phys. 69, 23 (2018)

52. Zeng, S.D., Migórski, S.: Noncoercive hyperbolic variational inequalities with applications to contact mechanics. J. Math. Anal. Appl. 455, 619-637 (2017)

53. Zeng, S.D., Migórski, S.: A class of time-fractional hemivariational inequalities with application to frictional contact problem. Commun. Nonlinear Sci. 56, 34-48 (2018) 